\title{
Progressive collapse simulation based on DEM for single-layer reticulated domes
}

\author{
Ye Jihong ${ }^{\text {巴 }}$, Qi Nian \\ Key Laboratory of Concrete and Pre-stressed Concrete Structures of the Ministry of \\ Education, Southeast University, Nanjing, China
}

\begin{abstract}
The conventional discrete element method (DEM) is usually applied to solve the elastic problems of granular materials. The DEM fibre constitutive model, which can consider the plasticity development of a cross-section, is derived, and then the fracture simulation algorithm for members is established based on the elastic-plastic analysis using this fibre model. Thus, the DEM is extended to study the continuum structures. Next, the proposed method is applied to the collapse simulation process of two single-layer reticulated dome test models. The simulation results, including the collapse process and the fracture location of joints, agree well with the shaking table test measured response. In addition, the displacement responses of representative nodes and axial force responses of representative members also are consistent with the experimental measured response, which demonstrates the accuracy and validity of this method. This study provides a new numerical analysis method for further investigation of the complicated mechanical behaviour-including large deformation, material nonlinearity and fractures — of large or super-large member structures.
\end{abstract}

Keywords: discrete element method; plasticity development; member fracture; single-layer reticulated dome; shake table collapse test; collapse simulation

\section{Introduction}

Structural progressive failure or collapse behaviour is a nonlinear process in which a body

\footnotetext{
Corresponding author: School of Civil Engineering, Southeast University, Nanjing 210018, China. Tel.: +86 2583795023. E-mail address: yejihong@seu.edu.cn (J.H. Ye).
} 
is forced from the continuum to the non-continuum (i.e., dynamic transformation between continuous and discontinuous analysis) with the occasional occurrence of member fracture. For steel structures, a fracture in the vicinity of a joint node would occur when the structures are subjected to strong earthquake or impact loads [1-2]. The conventional finite element method (FEM), derived from the continuum mechanics theory, is difficult to use when working with fractures and discontinuous problems. Therefore, the FEM, with special treatments and modifications, has been applied to fracture simulations. For instance, Lynn and Isobe [3] implemented the ASI-Gauss technique into finite element codes, including the fracture and contact problems of the elements, and then simulated the collapse of the World Trade Center under the aircraft impact. Recently, the vector-form intrinsic finite element (VFIFE) method, based on vector mechanics, has been proposed. This method has been successfully applied to the progressive collapse simulation of frames under earthquake and impact loads by Wang et al. [4], who analysed the nonlinear behaviour and collapse process of a plane steel frame exposed to fire caused by earthquake [5]. Moreover, the VFIFE has also been applied to the entire process simulation of earthquake-induced collapse of a mockup cable-stayed bridge [6]. These studies and applications by VFIFE are beneficial and provide a new method for numerical simulation of collapse.

The discrete element method (DEM) is essentially a discontinuum-based numerical method. The DEM has advantages in terms of simulating the problem involved such as large deformation, nonlinearity, and discontinuity [7-8]. The DEM discretizes a material by using rigid elements of simple shapes that are connected and interacted via springs. The behaviour of a member fracture can be achieved by defining the crack criterion of contact springs. In 
contrast to the FEM, the DEM is much more intuitive in behaviour mode when used to simulate the fracture, which can be seen as a natural process. Meanwhile, there are no special modifications or re-meshing for nonlinear analysis using DEM, and no iterations or stiffness matrices are integrated to solve the governing equation; thus, the DEM is more suitable to study the collapse of structures. A bonded-particle model (BPM) for rock was proposed by Potyondy and Cundall [9]. Once the maximum tensile or shear stress exceeds the ultimate strength, the parallel bond breaks; i.e., a crack is formed in the BPM. The numerical results from the literature [9-11] show that this model can well describe the progressive fracture process of the rock. Prochazka [12] extended the application of the discrete element method to fracture mechanics of rock bursts, and Sebastien et al. [13] adopted the method to model concrete beam submitted to dynamic loading. Moreover, a fracture model suitable for the spherical DEM is proposed based on the cohesive model, and the simulation of the laminated glass impact problem is developed, which agreed with the experimental phenomena [14]. In addition, the particle DEM is used to investigate the fracture mechanism of asphalt concrete from the mesoscale of material in the literature [15-16]. The initiation and propagation of cracking in asphalt concrete during the bending fracture process are analysed, and a comparison between the simulation and experimental results reveal similarity between them. To fully explore the potential of the two numerical methods, in the literature [17-19], there is an increasing interest in combining the FEM and DEM methods into a formulation that can apply the DEM in the simulation of large deformation or fracture regions, whereas other regions are simulated by the FEM. However, it is noted that these studies are focused on objects such as rocks and concrete and are used to simulate the fracture of quasi-brittle 
materials. Meanwhile, the constitutive equation of material is usually limited in the elastic range, and the particle elements in the DEM are arranged in multiple rows and columns, so they are unsuitable for spatial reticulated domes.

Single-layer reticulated domes, which are a typical example of a spatial structure, have been widely adopted in public buildings such as large gymnasiums and exhibition halls because of their beautiful form, ability to withstand reasonable loads, large span and effective cost. However, the collapse mode and failure mechanism of this type of structure during earthquake events are still under investigation [20-22]. Therefore, research on the collapse simulation analysis of single-layer reticulated domes under earthquake action by means of the numerical computational method has great theoretical significance and engineering value. In this paper, first, a fibre constitutive model in the DEM is proposed, which can consider the plasticity development of cross-sections. Based on the elastic-plastic analysis of structures with the fibre model, the algorithm considering member fracture is then established, and the solution flow is given. Finally, the proposed method is applied to the collapse process simulation of single-layer reticulated domes, and the validity is verified by the shaking table model test.

\section{Fibre constitutive model of the DEM}

In the FEM, compared with the plastic hinge model, the fibre beam-column element model can be refined to simulate the plasticity development of members and allow us to adopt different constitutive equations of materials for a cross-section; thus, it has been widely applied in structural engineering [23-27]. The principle of the fibre model can be described as follows: first, the section of a beam-column element is discretized into several fibres. Next, 
according to the plane section assumption, the mechanical behaviour of each fibre can be obtained easily based on the uniaxial stress-strain relation of the materials. We can then obtain the nonlinear hysteretic properties of the element section by integrating over all the fibres of the cross-section. Hence, the fibre model is able to consider exactly the complicated behaviour of members such as stiffness degradation, strength degradation and the interaction of the axial force and (uniaxial and biaxial) bending moment.

In this paper, the idea of the fibre model is extended to the DEM. The bond formed by two connected particles (i.e., spherical elements) is represented by the distributed springs as an equivalent. The "space beam element" comprises these distributed springs, and each spring corresponds to a fibre of the "beam element". Here, the "beam element" indicates the bond formed by two connected particles. The distance between the centres of two spheres is the length of the "beam element". The shapes and sizes of cross-sections of the "beam element" are consistent with the structural member itself.

Taking the space frame (Fig. 1) as an example, the cross-section of the beam and column of the frame is assumed to be a hollow circular pipe. The member of the beam or column is discretized into a row of spherical elements when the DEM model of the frame is created. Because the cross-section of a hollow circular pipe is used, the section of the "beam element" (i.e., the bond formed by two connected particles) is also the same. The length is the distance between the centres of two spheres, and the diameter and thickness of cross-section of the bond are identical to those of the member of the beam or column. Meanwhile, for convenient calculation, the location of fibres is assumed to be consistent with the 8 Gauss integral points of the pipe. Thus, the bond constitutive equation of the spherical elements can be determined 
according to the constitutive equation of each spring of the "beam element".

\subsection{Calculation of the fibre strain}

As shown in Figure 1, the section of the "space beam" at location $x$ along the longitudinal axis is subdivided into $n(=8)$ fibres. Each fibre is in a one-dimensional forcing state, and the fibre strain is obtained by the summation of the strain due to axial deformation and that due to bending curvature:

$$
\varepsilon=\varepsilon_{N}+\varepsilon_{M_{y}}+\varepsilon_{M_{z}}
$$

where $\varepsilon_{N}$ stands for the axial strain at the longitudinal axis, and $\varepsilon_{M_{y}}, \varepsilon_{M_{z}}$ stand for bending strain around the $y$ - and z-axis, respectively, and are computed as follows:

$$
\left\{\begin{array}{l}
\varepsilon_{M_{y}}=\kappa_{y} z_{i} \\
\varepsilon_{M_{z}}=\kappa_{z} y_{i}
\end{array}\right.
$$

where $\kappa_{y}, \kappa_{z}$ stand for the bending curvature around the $y$ - and $z$-axes, respectively; $y_{i}, z_{i}$ are the distance to the neutral $y$ - and $z$-axes of the cross-section for the $i^{\text {th }}$ fibre, respectively.

In the DEM, we can compute and obtain the displacement, velocity and angular velocity, etc., of each spherical element with the application of Newton's second law. As shown in Figure 1, at time $t$, the distance (i.e., length) between two sphere centres is denoted by $L$, and $\boldsymbol{n}$ is the unit normal vector directed along the length of the line. Next, the relative normal displacement increment, $\Delta U^{\mathrm{n}}$, and two tangent rotation increments, $\Delta \theta_{\tau^{y}}$ and $\Delta \theta_{\tau^{z}}$, of spheres A and B occurring over a time step of $\Delta t\left(=t_{i+1}-t_{i}\right)$ can be obtained by solving the governing equation of motion. Based on the plane section assumption, the incremental strain and curvature of a fibre at a time step of $\Delta t$ are given by 


$$
\left\{\begin{array}{l}
\Delta \varepsilon_{N}=\frac{\Delta U^{\mathrm{n}}}{L} \\
\Delta \kappa_{y}=\frac{\Delta \theta_{\tau^{y}}}{L} \\
\Delta \kappa_{z}=-\frac{\Delta \theta_{\tau^{z}}}{L}
\end{array}\right.
$$

where $\Delta \varepsilon_{N}$ stands for the axial strain increment; $\Delta \kappa_{y}, \Delta \kappa_{z}$ are bending curvature increments around the y- and z-axes, respectively.

Substituting Eq. (3) into Eq. (1) combined with Eq. (2) yields the following:

$$
\Delta \varepsilon=\Delta \varepsilon_{N}+\Delta \kappa_{y} z_{i}+\Delta \kappa_{z} y_{i}
$$

After the strain of each fibre in the cross-section of the "space beam" known, the stress of each fibre is then determined from the stress-strain constitutive relation, which finally enables us to achieve the elastic-plastic analysis by the DEM.

\subsection{Calculation of internal contact forces}

The increase in internal contact forces can be integrated over all the stress $\sigma$ of fibres in the cross-section:

$$
\left\{\begin{array}{l}
\Delta F^{\mathrm{n}}=\int \Delta \sigma \mathrm{dA}=\sum_{i=1}^{n} \Delta \sigma_{i} \cdot \mathrm{A}_{i} \\
\Delta M_{y}=\int \Delta \sigma \cdot z \mathrm{dA}=\sum_{i=1}^{n} \Delta \sigma_{i} \cdot z_{i} \cdot \mathrm{A}_{i} \\
\Delta M_{z}=\int \Delta \sigma \cdot y \mathrm{dA}=\sum_{i=1}^{n} \Delta \sigma_{i} \cdot y_{i} \cdot \mathrm{A}_{i}
\end{array}\right.
$$

where $\mathrm{A}_{i}$ is the area of the $i^{\text {th }}$ fibre; $\Delta F^{\mathrm{n}}$ is the normal contact force increment of the cross-section; and $\Delta M_{y}, \Delta M_{z}$ are bending moment increments around the $\mathrm{y}$ - and z-axes at a local coordinate, respectively.

Moreover, it is noted that there also exist the tangent components of contact force $F^{\tau}$ and contact twisting moment $M^{\mathrm{n}}$, in addition to the normal component of contact force $F^{\mathrm{n}}$ and 
contact bending moments $M_{y}, M_{z}$, when two spheres are in contact. However, in this study, the relations of shear contact force and deformation, and contact torsion and torsion angle are assumed to remain linear elastic. They are calculated by Eq. (6), according to the literature [28-29]:

$$
\Delta \mathbf{S}=\mathbf{K}_{e} \Delta \mathbf{U}
$$

where $\quad \Delta \mathbf{S}=\left[\begin{array}{llll}\Delta F_{y}^{\tau} & \Delta F_{z}^{\tau} & \Delta M^{\mathrm{n}}\end{array}\right]^{T}, \quad$ and $\Delta \mathbf{U}=\left[\begin{array}{ccc}\Delta U_{y}^{\tau} & \Delta U_{z}^{\tau} & \Delta \theta^{\mathrm{n}}\end{array}\right]^{T} ;$ $\mathbf{K}_{e}=\operatorname{diag}\left[-\frac{12 E I_{z}}{L^{3}}-\frac{12 E I_{y}}{L^{3}}-\frac{G J}{L}\right]$, where diag denotes the diagonal matrix.

\section{Algorithm considering member fracture}

\subsection{Fracture criteria}

Research on the fracture process for steel structures involves the material test and fracture mechanics, in which the refined structural model (e.g., 3-D solid model) is often very complicated. Here, we study the members' fracture behaviour from the macro point of view to ensure a certain level of accuracy. Basic assumptions are given as follows when the fracture simulation is carried out by the DEM:

1) Structural members are assumed to be ideal with no existing initial crack and defect;

2) The fracture criteria of members are established based on the material test;

3) The energy dissipation such as vibration and voice are not considered except for the damping dissipation;

4) The contact and collision behaviour of members after fracture is not considered.

In this study, based on the fibre model, a simple strain-dependent or stress-dependent fracture criterion at the material level is adopted to determine member fracture (see Fig. 1):

$$
\varepsilon_{k}^{t} \geq \varepsilon_{\mathrm{u}} \text { or } \sigma_{k}^{t} \geq \sigma_{\mathrm{u}}
$$


where $\varepsilon_{k}^{t}, \sigma_{k}^{t}$ are the strain and stress of the $k^{\text {th }}$ fibre at time $t$, respectively; and $\varepsilon_{\mathrm{u}}, \sigma_{\mathrm{u}}$ are the failure strain and stress, respectively.

If Eq. (7) is satisfied, the $k^{\text {th }}$ fibre (i.e., spring) is considered to be fractured and to have stopped working. Meanwhile, the stress of this fibre, $\sigma_{k}^{t}$, is set to zero.

The damage index of the section, $D^{t}$, is defined as

$$
D^{t}=\frac{\mathrm{N}_{\text {crack }}^{t}}{\mathrm{~N}}
$$

where $\mathrm{N}$ is the total number of fibres in a cross-section, and $\mathrm{N}_{\text {crack }}^{t}$ is the fractured fibre number $\left(\mathrm{N}_{\text {crack }}^{t} \leq \mathrm{N}\right)$ at time $t$.

As seen in Eq. (8): $0 \leq D^{t} \leq 1$. At the initial stage, the contact bond of spherical elements is intact, $D^{t}=0$. If all fibres in the section have fractured at some time $t, \mathrm{~N}_{\text {crack }}^{t} \leq \mathrm{N}$, so we can obtain $D^{t}=1$. Hence, the index $D^{t}$ clearly indicates the damage extent of a cross-section, which can describe the cumulative damage of the gradual failure process of members' sections of structures under loads.

\subsection{Fracture mode}

When the members have fractured, problems such as the crack of the contact bond of two adjacent spherical elements, separation of spherical elements and redistribution of structural internal force must be addressed and solved. For simplicity, the following hypotheses are assumed for fracture mode:

1) The members' fracture occurs only at the contact bond, but the crack of the spherical element itself is not considered;

2) The total number of spherical elements will remain unchanged before and after fracture;

3) Although the number of contact bonds will decrease and the topology relation of the 
members will change after fracture, the original spherical element and contact bond numbering will remain unchanged.

Taking the cantilever beam as an example, the DEM model of the beam, 11 spherical elements and 10 contact bonds are shown in Figure 2a. Under the vertical load $P$, the internal force of the section near the fixed end of the beam is the largest, so the strain (stress) of contact bond 1 first meets the fracture criterion and then will fracture. When contact bond 1 formed by spherical element 1 and element 3 has fractured, all springs will be removed such that the total number of contact bonds are reduced to 9 from 10 , but the total number of elements is still 11, as shown in Figure $2 b$.

In addition, after the contact bond has completely broken, the internal forces of the element at the crack location must be updated. According to the basic theory of the DEM, the resultant internal forces and moments of each spherical element are derived from the internal contact force and moment at the contact point of the two connected spherical elements. Thus, when the contact bond has broken and vanished, the contact force and moment of the section must be set to zero, thus obtaining the updated internal forces and external forces of the spherical element in the simulation at the next time step.

\subsection{Computational procedures}

According to Section 3.2, compared with the elastic-plastic analysis module by the DEM, only the fracture criteria subroutine and update of some parameter calculations are added into the computational procedures, but most of the other procedures still remain unchanged. Therefore, when the DEM is adopted to simulate member fracture, the subroutine of fracture can be transplanted into the original codes (i.e., elastic-plastic analysis). If the analysis is 
required, then we can call the subroutine, which can greatly improve the calculation efficiency.

We now define two status indexes:

1) The status index of fracture of the fibre integral point (i.e., spring) in a cross-section, which is denoted by Fibre_crack. At the initial time, each of the springs is intact, so Fibre_crack=1; if some spring has met the fracture criterion of Eq. (7) in the calculation, then Fibre_crack $=0$.

2) The status index of the crack of a cross-section (i.e., contact-bond), which is denoted by Damage. At the initial time, each of contact bonds is intact, so Damage=1; if some contact bond has reached the fracture criterion of Eq. (8), $D^{t}=1$ in the calculation, so Damage=0. When Damage $=0$, the total cross-section is cracked, which means that the contact bond between the two adjacent spherical elements has fractured, and then the spherical elements are separated.

Finally, based on the foregoing analysis, the calculation flowchart for simulating the collapse of structures considering member fracture by the DEM is given in Figure 3.

\section{Verification and collapse simulation of single-layer reticulated domes under earthquake}

In the literature [30], research on collapse via shaking table tests of single-layer reticulated domes has been performed. Three scale models of Kiewitt-6 single-layer domes were designed, and the geometric similarity ratio of the model and the prototype is $1: 10$. In this study, the proposed DEM considering member fracture is applied to the collapse simulation of two test models and compared with experimental results. 
Figure 4 shows the structural arrangement of the two models. Model 1 and Model 2 have the same topological configuration with a span of $7.5 \mathrm{~m}$ and a rise-to-span ratio of 0.5 . There are 265 nodes and 744 members. The 24 fixed supports are arranged alternately along the periphery of the model. In Model 1, there are six predetermined weak links located on each of the six radial main ribs. The members in those weak links are high-strength stainless steel tubes with a cross-section of $\Phi 8 \times 0.5 \mathrm{~mm}$ (represented by dotted lines), as shown in Figure 4(a). The other members are high-strength stainless steel tubes with a cross-section of $\Phi 14 \times 0.6 \mathrm{~mm}$. In Model 2, all members are high-strength stainless steel tubes with a cross-section of $\Phi 14 \times 0.6 \mathrm{~mm}$. The elastic modulus and the yield strength of the steel were obtained by a standard material performance test, as given in Table 1. To satisfy the requirements of the scale similarity, masses weighing $5.61 \mathrm{~kg}$ are fixed on the nodes of the weak links; the weight on all other nodes is $10.43 \mathrm{~kg}$ in Model 1, and the weight of the mass fixed on each node is $10.43 \mathrm{~kg}$ in Model 2.

\subsection{Collapse simulation and verification of Model 1}

\subsubsection{The domain meshing of Model 1}

To conserve computing resources, the small deformation region is simulated by the FEM, and the other region with large deformation or member bending or separation from nodes is simulated by the DEM. The interface in the coupled DEM/FEM model is treated by penalty function whose scale factor equals to 32.0 [31]. As shown in Figure 5, a Beam4 element is meshed in each member within the FEM domain. In the DEM domain, the welded sphere is treated as a separate spherical element, and each member is discretized into 5-10 spherical elements with radii ranging from 45 to $65 \mathrm{~mm}$. There are 3678 spherical elements and 3916 
bonding elements in the coupled model, along with 54 contact point pairs existing in the interface.

\subsubsection{The fracture simulation of the welded seam}

When the domes are analyzed by the FEM using beam elements, the size of the welded spherical joint itself is usually ignored, so the fracture phenomena between members and spherical joints cannot be simulated. However, in this study, the member is modelled by a row of DEM spheres, and the welded joint is treated as a separate sphere. Therefore, regardless of whether the fracture will occur in the welded seam between members and joints during the test, the discrimination of fracture can be conveniently carried out based on the criterion in section 2.1.

When the test models of the dome were fabricated, shielded metal arc-welding was used on both members and joint nodes. The welded spherical joints were made of Q235 steel, corresponding to electrode E43. The young modulus of the welded seam is the same as that of stainless steel tube. The experimental results in the literature [32] showed that the average ultimate failure stress of end welded seams was $560 \mathrm{MPa}$ when electrode E43 was used for welding Q235 steel. Therefore, the value of failure stress in simulating the fracture of the welded seam by the DEM fibre model is $560 \mathrm{MPa}$ in this paper.

\subsubsection{Verification of the collapse process between the test and simulation results}

(1) Description of the collapse process for testing Model 1

The El-Centro seismic wave is input along the $\mathrm{X}$ direction (see Fig. 4). The seismic wave was gradually improved with an increment of 200 gal of the peak ground acceleration (PGA). First, the vibration of the dome was inconspicuous when the PGA was small. With increasing 
PGA, the vibration of the weak links in the structure became more apparent and more severe than in other regions. Because all members were made of high-strength stainless steel, no noticeable structural deformation occurred before the PGA reached 1,200 gal. As the PGA reached 1,268 gal, some members with a cross-section of $\Phi 8 \times 0.5 \mathrm{~mm}$ bent in the weak zones of the second and third rings at the bottom of the model (Fig. 6a). When the PGA reached 1,534 gal, some joints in the weak links depressed suddenly, and the members connected to these joints experienced obvious bending deformation (Fig. 6b). Meanwhile, several members separated from the spherical joints (Fig. 6c), resulting in partial collapse of the model.

(2) Simulated results of the collapse process

An identical seismic wave was input when the numerical simulation was performed. Rayleigh damping was used with a damping ratio of $2 \%$. The first order natural frequency is obtained from white noise sweep results [30]. The time step of the coupled model was $6 \times 10^{-5} \mathrm{~s}$. Figure 7 shows the simulated failure process of Model 1 by the coupled DEM/FEM method. As shown in the figure, when the PGA was less than 1100 gal, the vibration of the dome was very small, and the structure had almost no deformation. As the PGA reached 1100 gal, although some members with a cross-section of $\Phi 8 \times 0.5 \mathrm{~mm}$ bent in the weak zones of the second and third rings at the bottom of the model, the nodal displacement and the bending amplitude were both small (Fig. 7a). When the PGA reached 1200 gal, owing to the high-strength and brittleness of the stainless steel, a few sections of welded seam had satisfied the fracture criterion, resulting in the members (i.e., $\Phi 8 \times 0.5 \mathrm{~mm}$ ) departing from the welded spherical joint. However, the number of fractures was small, and they were mainly focused in the location between the members and their corresponding joints, which experienced obvious 
bending deformation in the previous loading case (Fig. 7b). Afterwards, when the PGA reached 1400 gal, the number of members departing from joints at the weak zones increased. Meanwhile, the displacement of the corresponding joints increased significantly, and some members had been out of the structure and then initiated free motion (Fig. 7c). When the PGA continued to increase to 1600 gal, from the second to fifth rings, many more members departed from the welded spherical joint at the weak zones, but all other members remained intact (Fig. 7d).

To further investigate the detailed failure mechanism of Model 1, the dynamic responses of some nodes and members during the collapse process were track recorded. Several representative nodes and members selected from one-third of the model are numbered and marked in Figure 8. Figure 9 and Figure 10 are the displacement time history curves of the representative nodes when the PGA is 1600 gal. It can be found that the displacement amplitudes of Node 156 and Node 163 were very small at the initial time, and the vibration of the structure was obvious as the peak value of the earthquake wave increased. At approximately 1.7 s, Node 163 first departed from its members, resulting in the node displacement being suddenly large (achieved $260 \mathrm{~mm}$ ). The total horizontal displacement of node 163 reaches $339 \mathrm{~mm}$. Afterwards, Node 156 also departed from its members. Although Node 128 was located in the weak zones, with the seismic wave input along the horizontal direction, fracture between Node 128 and its connecting members did not occur; similarly, for Node 206, which was not located in the weak zones, the amplitude of its vibration was always very small during the whole process (no more than $7 \mathrm{~mm}$ ).

Figure 11 and Figure 12 show the axial force time history curves of the representative 
members when the PGA is 1600 gal. It can be found that the axial force amplitude of Member 395, Member 449 and Member 396, which are located in the weak zones, are large, with a maximum near $3000 \mathrm{~N}$. However, the axial force of Member 119, which is located in the same main rib, is smaller than that of Member 395. Because of the fracture of Node 156 and its linking members, Member 395, Member 449 and Member 396 departed from the structure after $3.8 \mathrm{~s}$, resulting in the axial force of all of those members being dramatically reduced to zero. During the whole loading process, the axial force of both Member 119 and Member 463 only fluctuated because the members and nodes in this area were always intact. At the later stage, as the seismic amplitude gradually decreased, the axial force of the members (i.e., Member 119, 463) decreased with passing time.

\subsection{Collapse simulation and verification of Model 2}

\subsubsection{Description of the collapse process for Model 2}

The test loading process of model 2 was the same as that of Model 1. Initially, when the peak value of the earthquake wave was small, the structure had almost no deformation. As the PGA increased to 1573 gal, the diagonal members near the supports began to bend (Fig. 13b). With the PGA increased, the diagonal members located in the first and second rings (from bottom to top) bended one by one, and the structural displacement increased gradually. As the PGA reached 2108 gal, the number of bending diagonal members in the first and second rings (from bottom to top) continued to increase; moreover, some members and spherical joints separated (Fig. 13c). Afterwards, when the PGA increased to 2268 gal, many more members and spherical joints located in the first and second rings departed from the dome, resulting in total structure falling down (Fig. 13d). 


\subsubsection{Simulated results of the collapse process}

The method to establish a numerical simulation model to test Model 2 is similar to that of Model 1. In other words, a Beam4 element is meshed in each member within the FEM domain. In the DEM domain, the welded spherical joint is treated as a separate spherical element, and each member is discretized into 5-10 spherical elements with radii ranging from 45 to $65 \mathrm{~mm}$. There are 2479 spherical elements and 2854 bonding elements in the coupled model, along with 78 contact point pairs existing in the interface.

Rayleigh damping is still employed with a damping ratio of $2 \%$. The first order natural frequency is obtained from white noise sweep results [30]. The time step is $6 \times 10^{-5} \mathrm{~s}$. In addition, although all members are made of high-strength stainless steel tubes with a cross-section of $\Phi 14 \times 0.6 \mathrm{~mm}$ in Model 2, the welded hollow spherical joints were made of Q235 steel, using electrode E43. As stated in section 4.1.2, the value of failure stress in simulating the fracture of the welded seam with the DEM fibre model is $560 \mathrm{MPa}$.

Figure 14 shows the simulated collapse process of Model 2. As shown in the figure, when the PGA was less than 1200 gal, the total structure had almost no deformation. When the PGA reached 1600 gal, a small number of diagonal members located in the bottom ring began to bend (Fig. 14a). As the PGA increased to 1800 gal, because a few sections of welded seam had satisfied the fracture criterion, a minuscule number of bending diagonal members departed from the welded spherical joints (Fig. 14b). With increasing PGA, the diagonal members located in the first and second rings (from bottom to top) bended one by one. When PGA reached 2100 gal, approximately half of the diagonal members in the first ring near the supports departed from the joints, and the structural displacement increased significantly (Fig. 
14c). However, the structure did not collapse in this case. When the PGA increased to 2300 gal, almost all members in the bottom ring departed from the joints and fractured. Meanwhile, some members in the second ring also departed from the joints. Consequently, the structure cannot achieve its ultimate bearing capacity owing to the loss of support from the members in the bottom rings; thus, the dome totally fell down under the gravity and earthquake action (Fig. 14c).

Figure 15 shows the displacement time history curves of the representative nodes (see Fig. 8) when the PGA is 2300 gal. It can be found that the displacement amplitudes of Node 156 and Node 163 were very small (a few millimetres) at the initial time. The structure vibrated significantly as the main shock of the earthquake wave arrived, and the number of fractures that occurred between the bottom members and their connecting joints increased. At approximately $2.2 \mathrm{~s}$, the structure collapsed totally, and the upper part moved downward with the rigid-body motion, resulting in abrupt increase in the nodal displacement (achieved at the metre scale). Figure 16 shows the axial force time history curves of the representative members when the PGA is 2300 gal. Because the peak value of the earthquake wave was very large in this case and the steel tubes were high-strength stainless, before $2.2 \mathrm{~s}$, the axial force amplitude of Member 395, Member 449 and Member 396 are large, with a maximum near 5000 N. However, the axial force of those members decreased when the structure collapsed (i.e., after $2.2 \mathrm{~s}$ ). At the later stage, as the seismic amplitude gradually decreased, the axial force of those members decreased with passing time. During the entire loading process, because the members in the third ring and those above it maintained their links with the joints and remained intact, the axial force of those members (i.e., Member 395, Member 449 and 
Member 396) only fluctuated and steadily decreased rather than dramatically decreasing to zero.

By comparing the collapse processes of Model 1 and Model 2, the collapse mechanism of Model 2 under earthquake action can be summarized as follows: as the PGA increased gradually, the diagonal members in the first and second rings near the supports first began to bend, and then almost all of the members in the first and second rings bent as the members separated from their connecting joints. Finally, the structure totally collapsed from the loss of its ultimate bearing capacity. Model 1 contained weak links, so the dome did not possess uniform structural rigidity. As seen in the simulation results, the members bent and departed from joints only in the weak zones, so the structure experienced partial collapse. In this study, the simulation results of the DEM approach considering member fracture agreed well with the model test phenomena. Moreover, according to the displacement responses of representative nodes and axial force responses of representative members, the experimental phenomena were also explained well. The validity of the proposed method was verified.

\section{Conclusions}

(1) The conventional DEM is usually applied to solving the elastic problems of granular materials. To simulate the complicated mechanical behaviour (i.e., large deformation, material nonlinearity and fractures, etc.) that occur in the collapse process of structures, the DEM is extended to study the continuum structures. The DEM fibre constitutive model, which can consider the plasticity development of sections, is derived, and the fracture simulation algorithm based on the elastic-plastic analysis using the fibre model is then established.

(2) The proposed method is applied to the collapse simulation process of single-layer 
reticulated dome models. Compared with the shaking table test, it is observed that the simulation results, including the collapse process and the fracture location of joints, agree well with the experimental phenomenon. In addition, the displacement responses of representative nodes and axial force responses of representative members also explain the experimental phenomena well, which demonstrates the accuracy and validity of this method.

(3) The present work demonstrates that the DEM can overcome the difficulties involved in simulating the problem using the conventional numerical method, such as strong nonlinearity and discontinuity. Compared with the FEM, no iterations are necessary to follow nonlinearity laws, and no special treatments are formed or solved in the DEM. The DEM method is more efficient in computation. This study lays the foundation for further refining investigation of the failure mechanism and entire-process simulation of the collapse of the structure and also for providing a new numerical analysis measure.

\section{Acknowledgments}

This work is supported by the National Science Foundation for Distinguished Young Scholars of China (Grant No. 51125031).

\section{References}

[1] Mahin SA. Lessons from damage to steel buildings during the Northridge earthquake [J]. Engineering Structures, 1998, 20: 261-270. 
[2] Rodgers J, Mahin SA. Effects of Connection Fractures on Global Behavior of Steel Moment Frames Subjected to Earthquake [J]. Journal of Structural Engineering ASCE, 2006, 132(1): 78-88.

[3] Lynn KM, Isobe D. Finite element code for impact collapse problems of framed structures [J]. International Journal for Numerical Methods in Engineering, 2007, 69: 2538- 2563.

[4] Wang CY, Wang RZ, Tai KC. Numerical Simulation of the Progressive Failure and Collapse of Structure under Seismic and Impact Loading [C]. 4th International Conference on Earthquake Engineering, Taipei, Taiwan, 2008, p.84-90.

[5] Lien KH, Chiou YJ, Wang RZ et al. Vector Form Intrinsic Finite Element analysis of nonlinear behavior of steel structures exposed to fire [J]. Engineering Structures, 2010, 32(1): 80-92.

[6] Duan YF, He K, Zhang HM et al. Entire-Process Simulation of Earthquake-Induced Collapse of a Mockup Cable stayed Bridge by Vector Form Intrinsic Finite Element Method [J]. Advances in Structural Engineering, 2014, 17(3): 347-360.

[7] Cundall PA, Strack ODL. A discrete numerical model for granular assemblies [J]. Geotechnique, 1979, 29(1): 47-65.

[8] Axel RT, Zoltan O. Discrete element analysis of a stone masonry arch [J]. Mechanics Research Communications, 2009, 36: 469-480.

[9] Potyondy DO, Cundall PA. A bonded-particle model for rock [J]. International Journal of Rock Mechanics and Mining Sciences, 2004, 41(8):1329-1364.

[10]Zhang XP, Wong LNY. Cracking processes in rock-like material containing a single flaw under uniaxial compression: A numerical study based on parallel bonded-particle model 
approach [J]. Rock Mechanics and Rock Engineering, 2012, 45(5): 711-737.

[11]Mohammd SA, Vamegh R, Giovanni B. A bonded particle model simulation of shear strength and asperity degradation for rough rock fractures [J]. Rock Mechanics and Rock Engineering, 2012, 45(5): 649-675.

[12]Procbazka PP. Application of discrete element methods to fracture mechanics of rock bursts [J]. Engineering Fracture Mechanics. 2004, 71(4): 601-618.

[13] Sebastien H, Frederic VD, Laurent D. Discrete element modeling of concrete submitted to dynamic loading at high strain rates [J]. Computers and Structures. 2004, 82(29): 2509-2524.

[14] Gao W, Zang MY. The simulation of laminated glass beam impact problem by developing fracture model of spherical DEM [J]. Engineering Analysis with Boundary Elements, 2014, 42: 2-7.

[15]Chen J, Wang LB, Huang XM. Micromechanical modeling of asphalt concrete fracture using a user-defined three-dimensional discrete element method [J]. Journal of Central South University, 2012, 19(12): 3595-3602.

[16]Kim H, William GB. Discrete fracture modeling of asphalt concrete [J]. International Journal of Solids and Structures. 2009, 46: 2593-2604.

[17] Onate E, Rojek J. Combination of discrete element and finite element methods for dynamic analysis of geomechanics problems [J]. Computer Methods in Applied Mechanics and Engineerig, 2004, 193(27): 3087-3128.

[18] Azevedo NM, Lemos JV. Hybrid discrete element/finite element method for fracture analysis [J]. Computer Methods in Applied Mechanics and Engineerig, 2006, 195(33): 
4579-4593.

[19]Rousseau J, Frangin E, Marin P. Damage prediction in the vicinity of an impact on a concrete structure: a combined FEM/DEM approach [J]. Computers \& Concrete, 2008, 5(4): 343-358.

[20] Shen, SZ, Zhi XD. Failure mechanism of reticular shells subjected to dynamic actions [C]. Proc 4th Int Conf on Advances on Steel Structure. Vol. I, Elsevier, London. 2005: 69-82.

[21] Ye JH, Liu WZ. Research on failure scenarios of domes based on form vulnerability [J]. Sci China Ser E, 2011, 54(11): 2834-53.

[22]Qi L, Shao YH, Huang ZW et al. Dynamic damage criterion and damagemode for single layer lattice shell [J]. Journal of Constructional Steel Research, 2014, 99: 102-110.

[23] Spacone E, Filippou FC, Taucer FF. Fibre beam-column model for non-linear analysis of R/C frames: Part I. Formulation [J]. Earthquake Engineering and Structural Dynamics, 1996, 25(7): 711-725.

[24] Spacone E, Filippou FC, Taucer FF. Fibre beam-column model for non-linear analysis of R/C frames: Part I. Applications [J]. Earthquake Engineering and Structural Dynamics, 1996, 25(7): 727-742.

[25]Richard LJY, Chen H. Explosion and Fire Analysis of Steel Frames Using Fiber Element Approach [J]. Journal of Structural Engineering ASCE, 2004, 130(7): 991-1000.

[26]Chen SC, Lu XZ, Ren AZ. Nonlinear analysis of multi-story concrete frame under fire with fiber beam model [J]. Journal of Building Structures, 2009, 30(6): 44-53. (in Chinese)

[27]Wang Q, Song XD, Hao ZH. Numerical simulation of failure process of RC columns 
based on fiber beam element in ABAQUS [J]. China Civil Engineering Journal, 2014, 47(12): 16-27. (in Chinese)

[28]Qi N,Ye JH. Geometric nonlinear analysis with large deformation of member structures by discrete element method [J]. Journal of Southeast University (Natural Science Edition), 2013, 43(5): 917-922. (in Chinese)

[29]Qi N, Ye JH. Discrete element and finite element methods for dynamic analysis of geomechanics problems [J]. Applied Mechanics and Materials, 2014, 638-640: 1716-1719.

[30] Ye JH, Pan R. Shaking table test on collapse process of single-layer spherical shells [J]. Journal of Building Structures, 2013, 34(4): 81-90. (in Chinese)

[31]Qi N. Coupled DEM/FEM method and its application in collapse simulation of reticulated shells [D]. Nanjing: Southeast University, 2015. (in Chinese)

[32]Chen SF. The Design Principle of Steel Structures [M]. Science Press. Beijng, China. 2011. 
Table 1. The material properties of the test models

\begin{tabular}{cccc}
\hline & & Elastic & Yield \\
Type & Specifications & modulus & stress \\
& & $(\mathrm{GPa})$ & $(\mathrm{MPa})$ \\
\hline Stainless steel & $\Phi 8 \times 0.5 \mathrm{~mm}$ & 198 & 884 \\
tubes & $\Phi 14 \times 0.6 \mathrm{~mm}$ & 191 & 864 \\
\hline
\end{tabular}




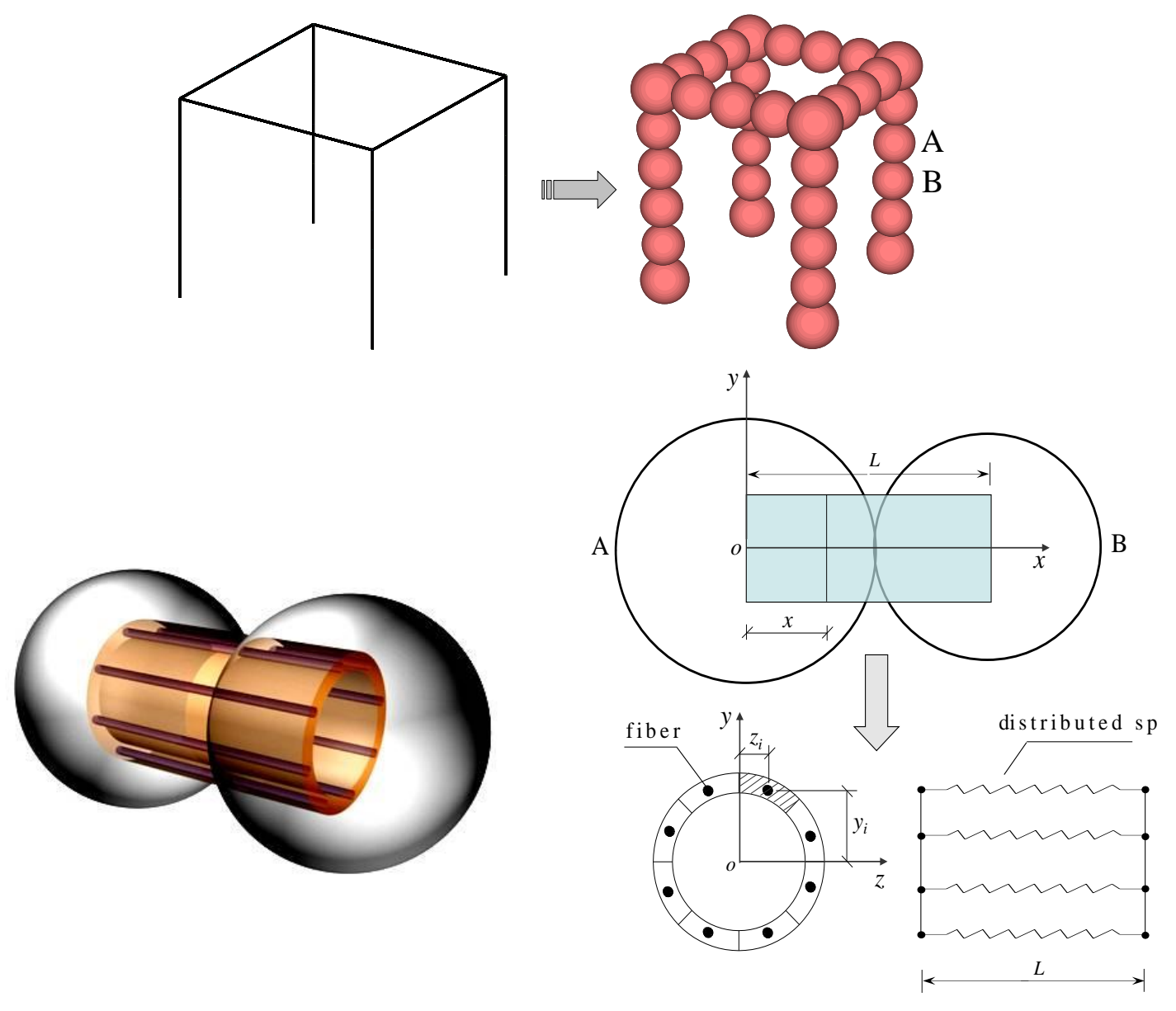

Fig. 1
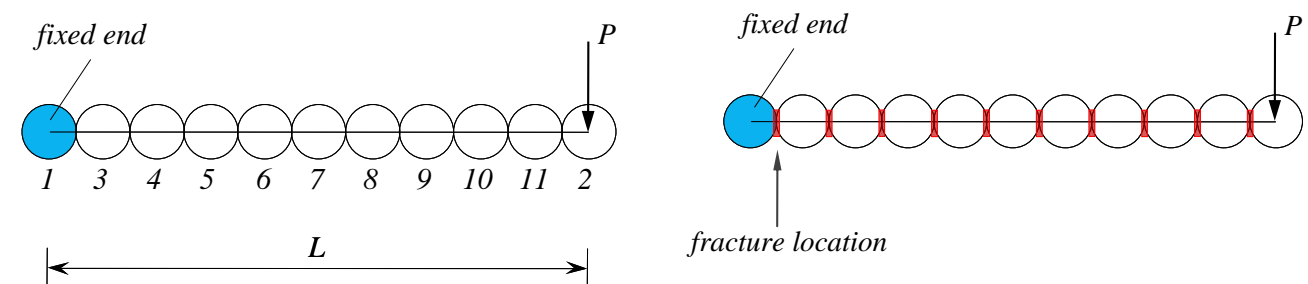

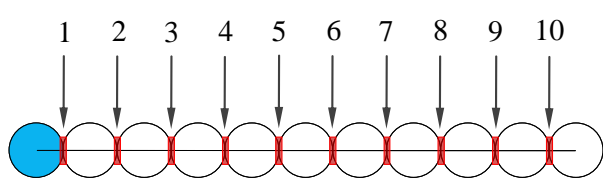

(a)

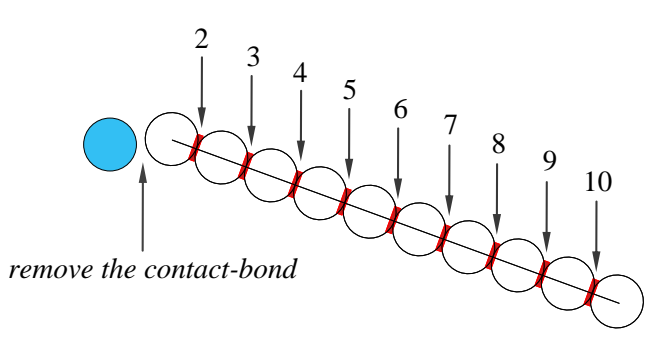

(b)

Fig. 2 


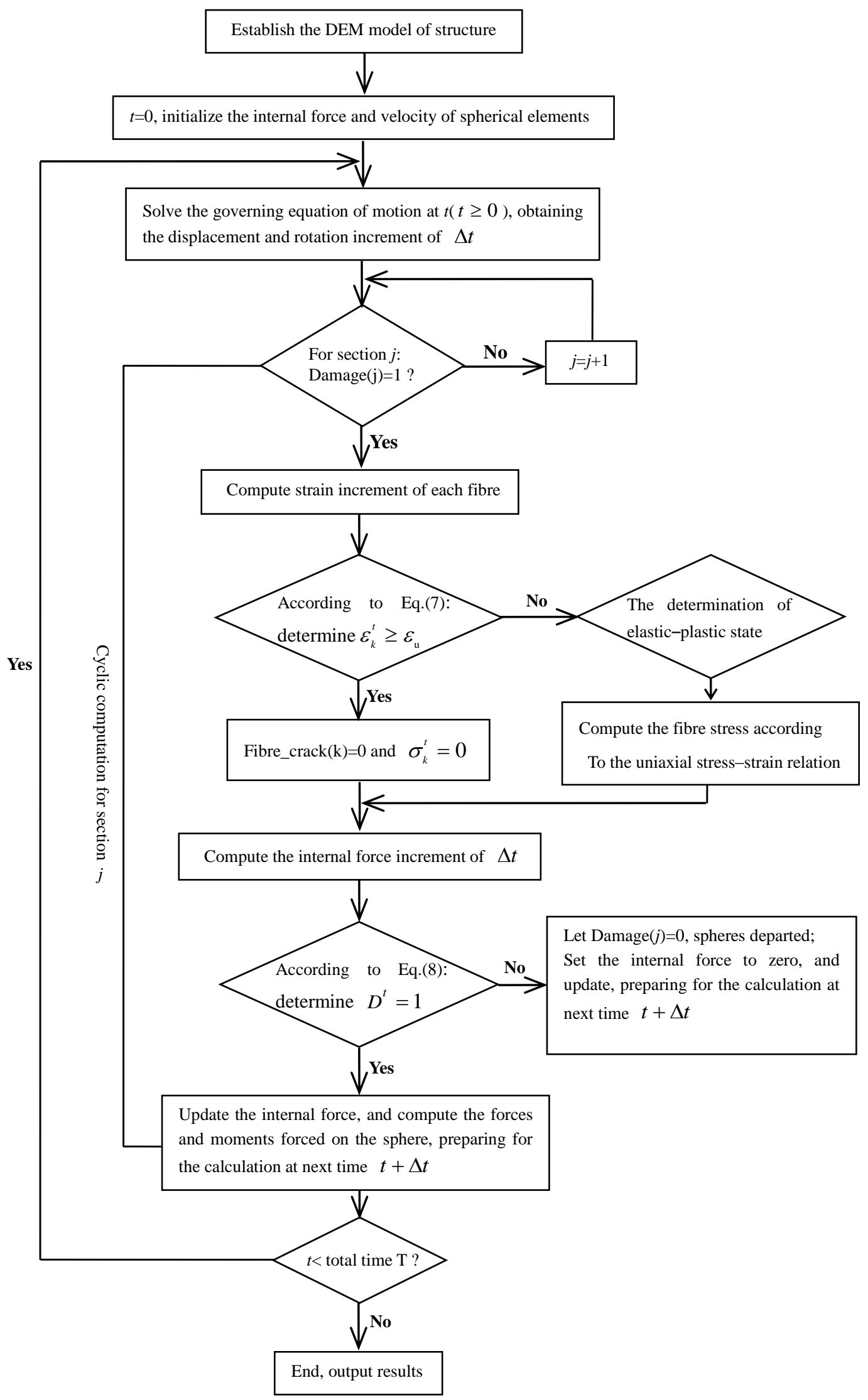

Fig. 3 


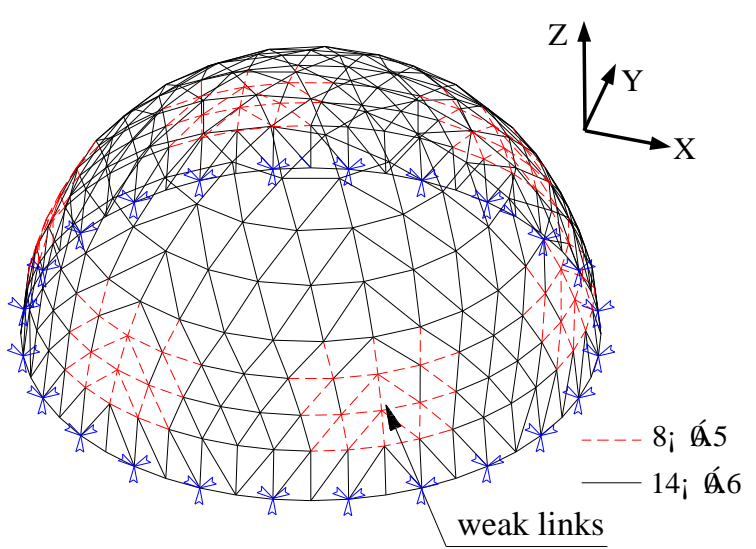

(a)

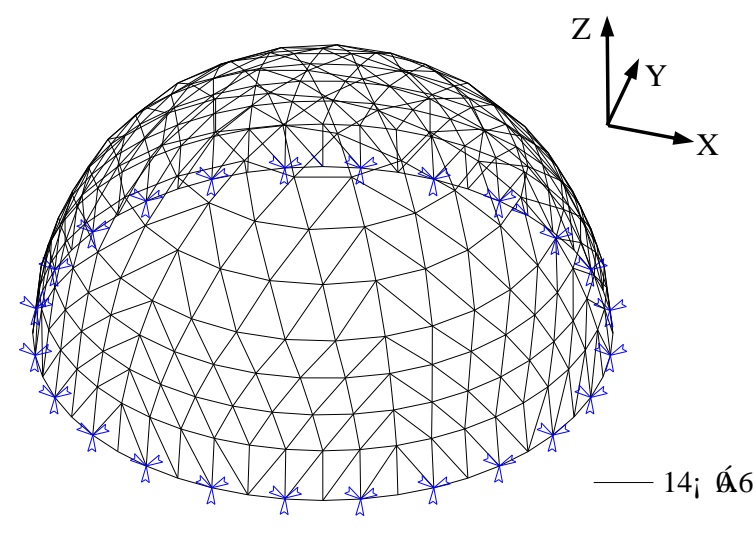

(b)

Fig. 4

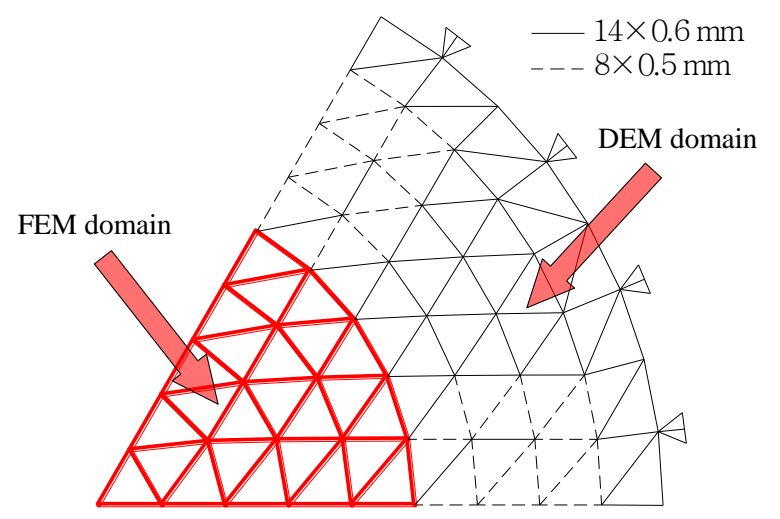

Fig. 5

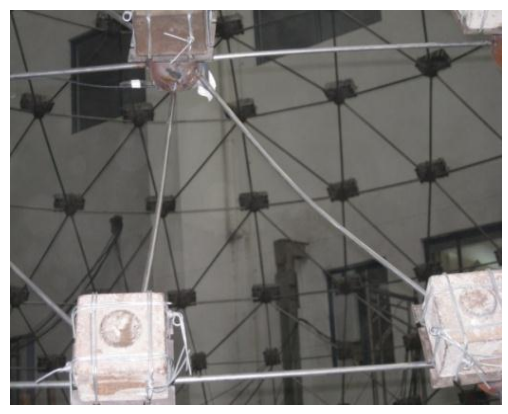

(a)

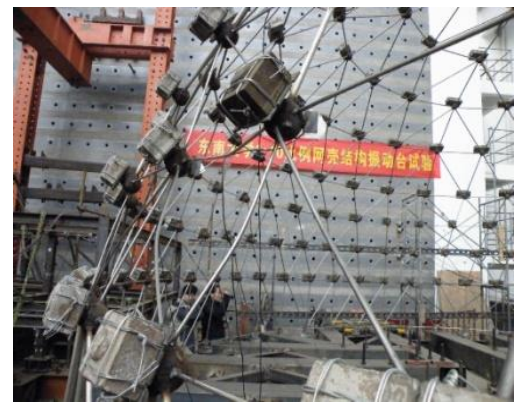

(b)

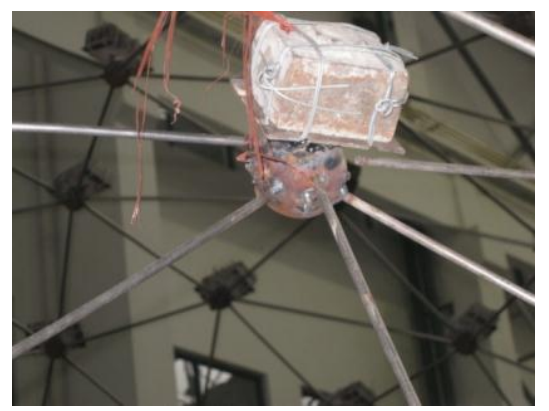

(c)

Fig. 6 


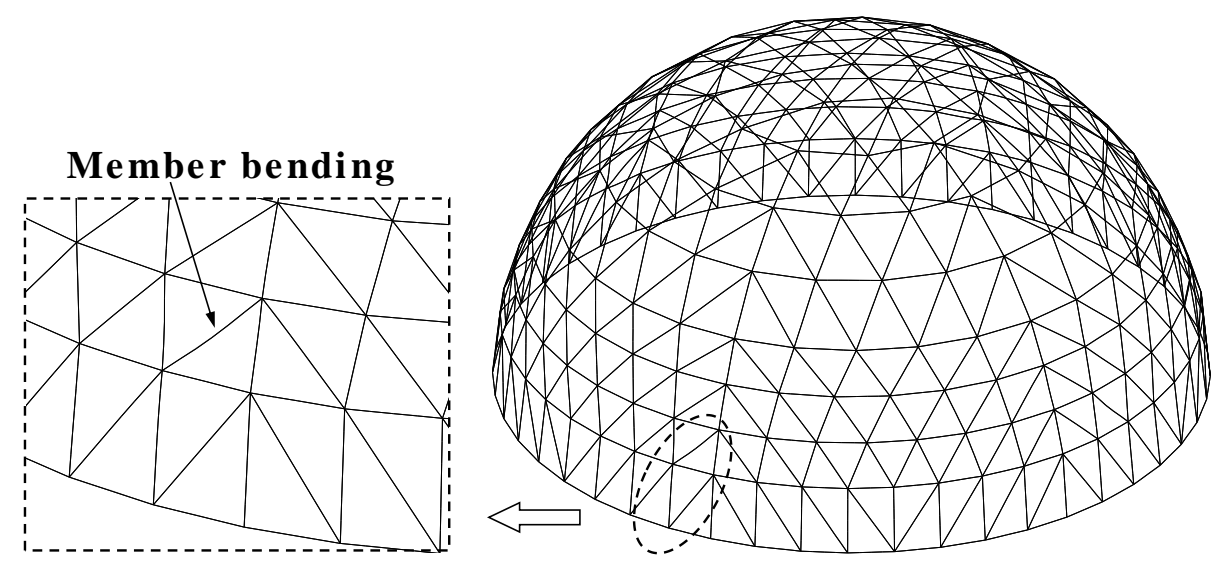

(a)

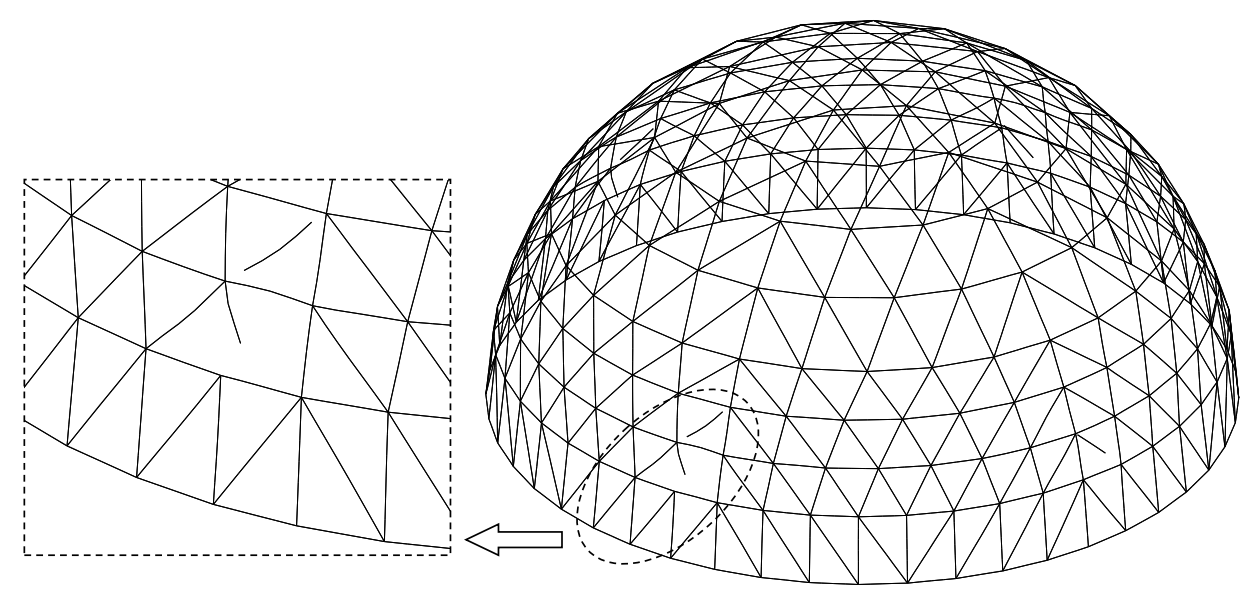

(b)

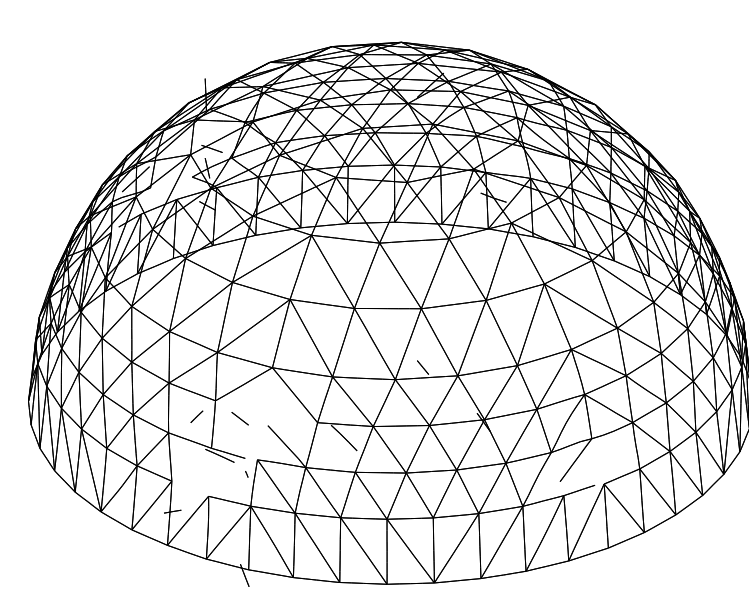

(c)

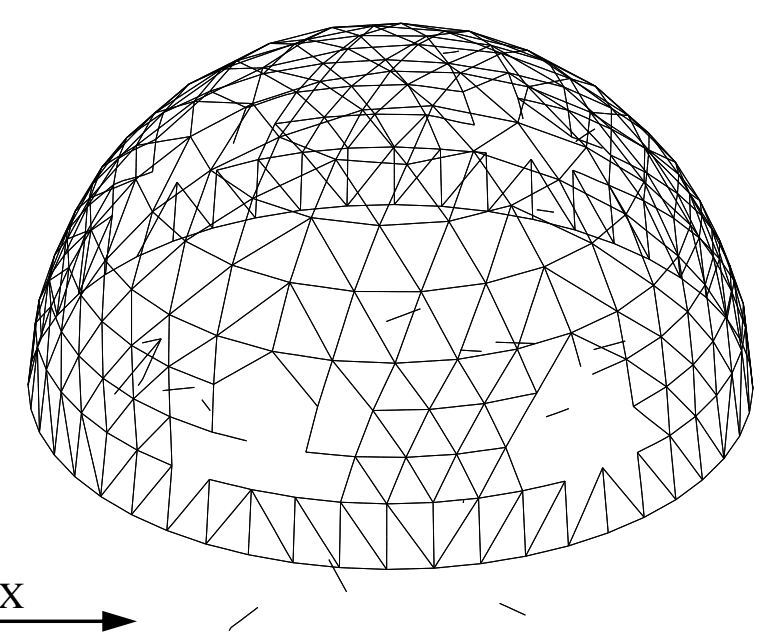

(d)

Fig. 7 


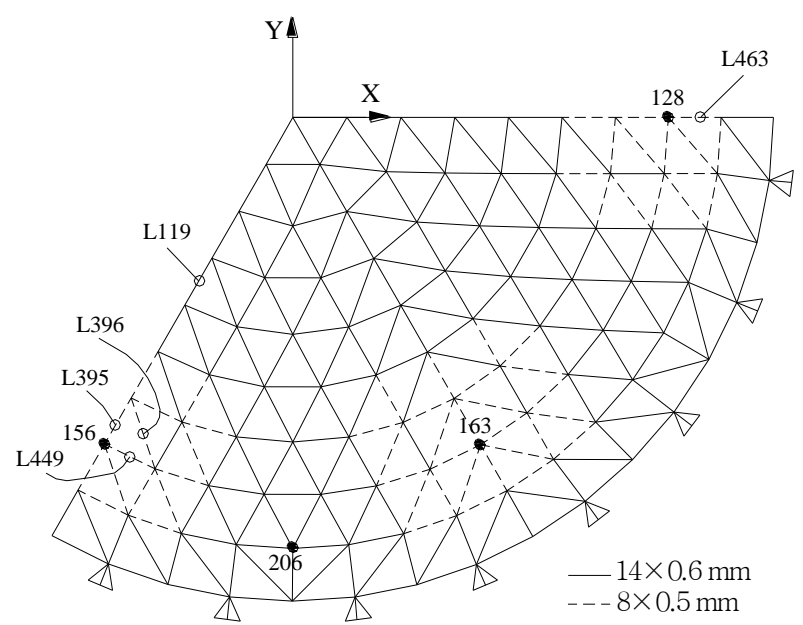

Fig. 8

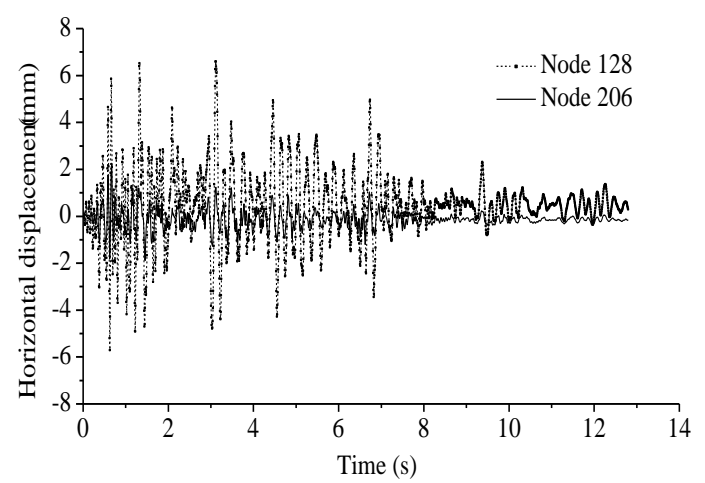

Fig. 9

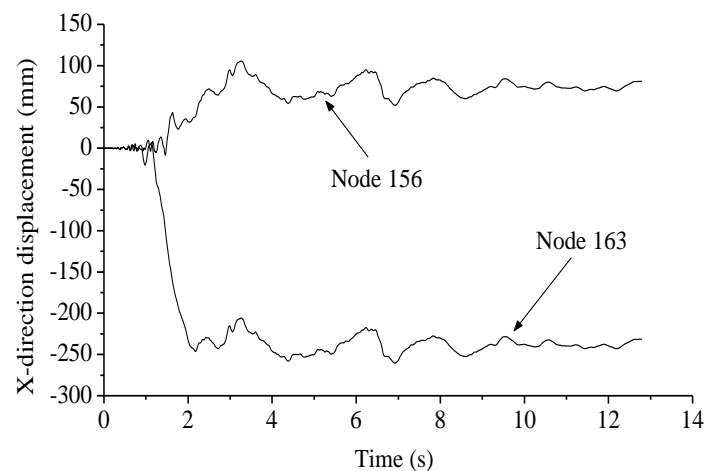

Fig. 10 


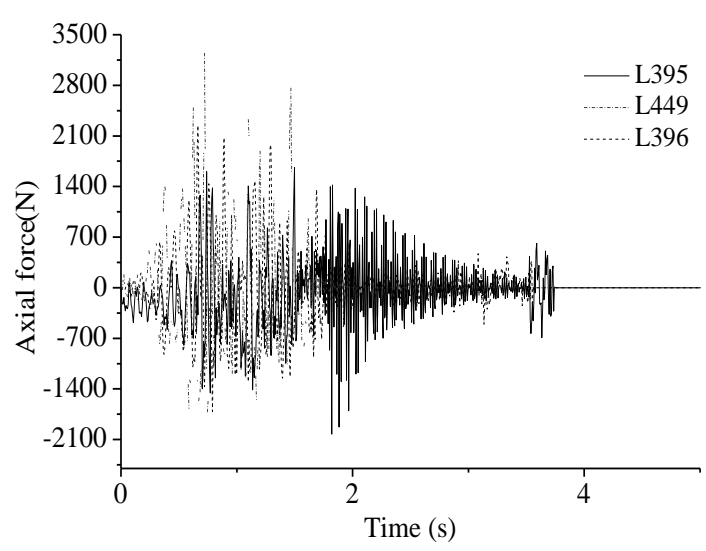

Fig. 11

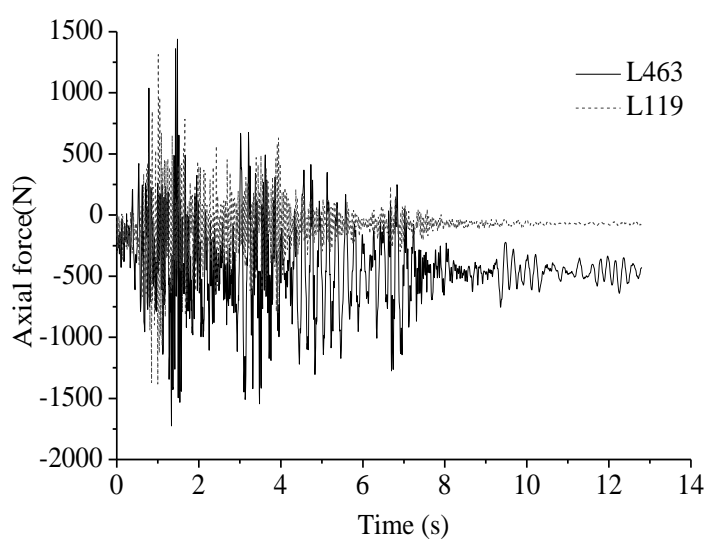

Fig. 12

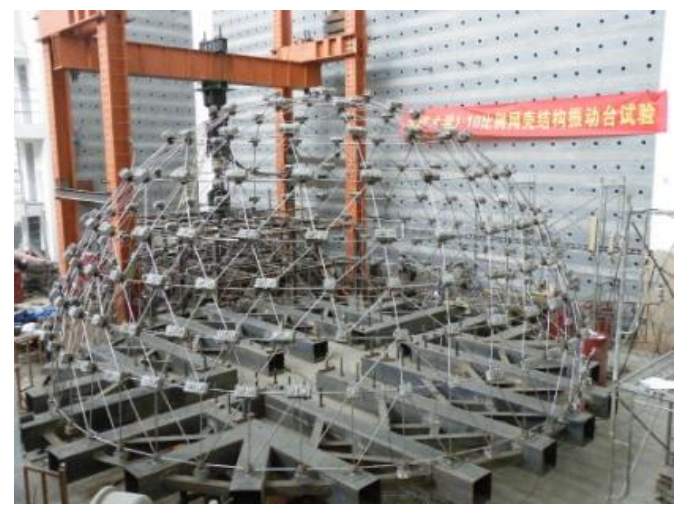

(a)

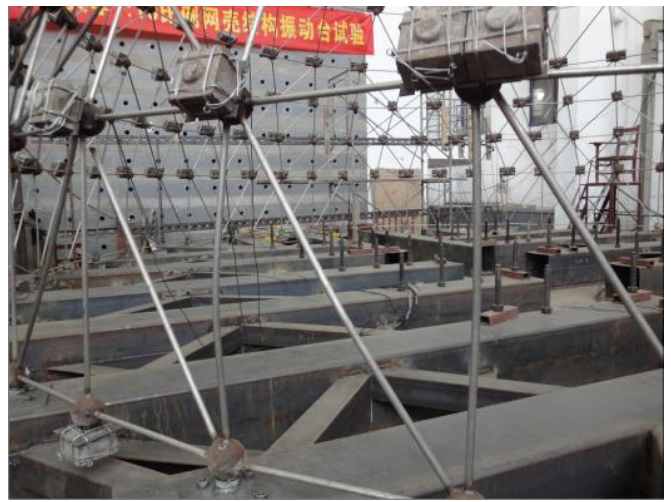

(b) 


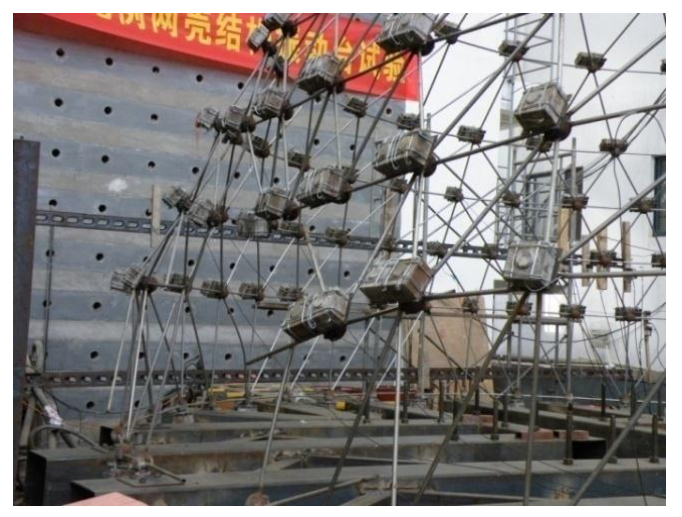

(c)

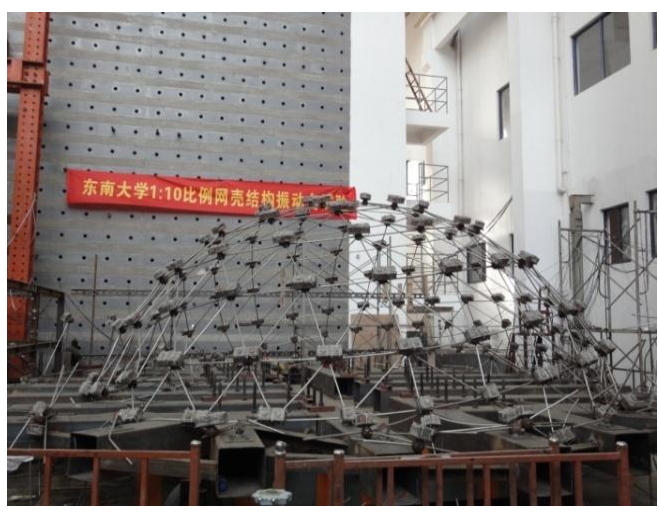

(d)

Fig. 13
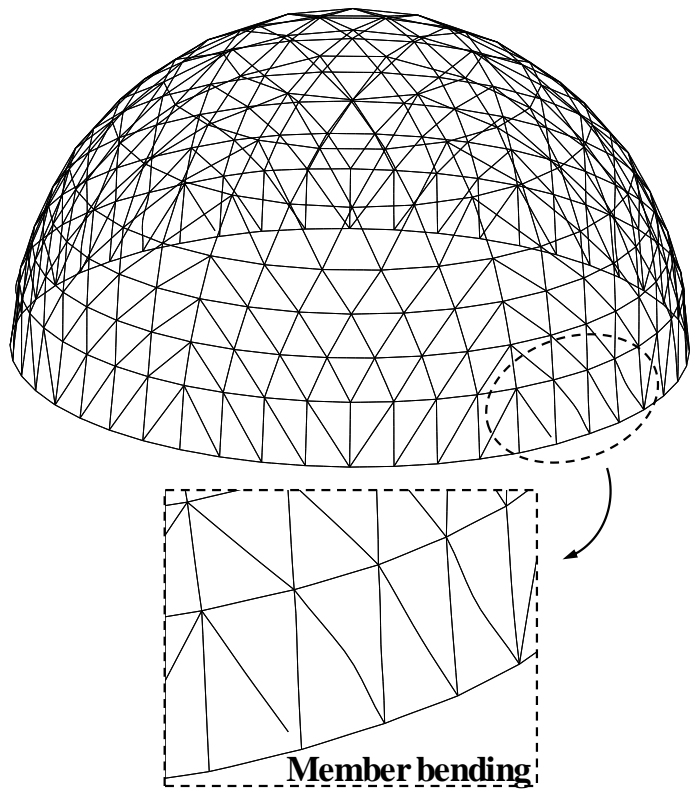

(a)

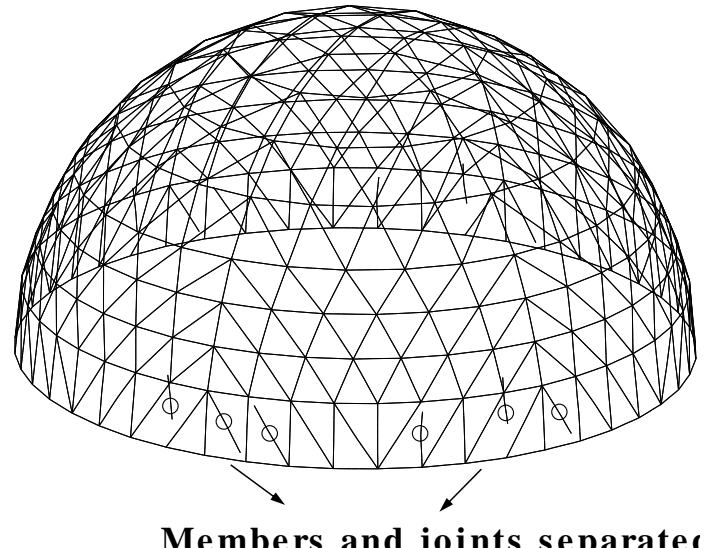

(b)

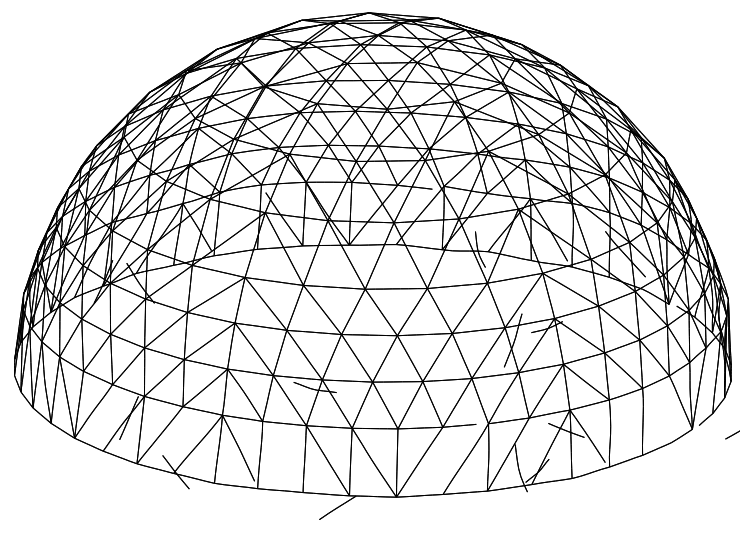

(c)

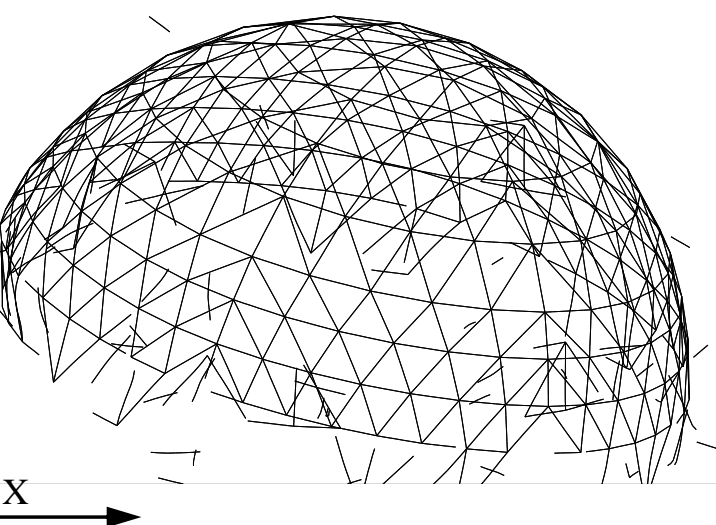

(d) 
Fig. 14

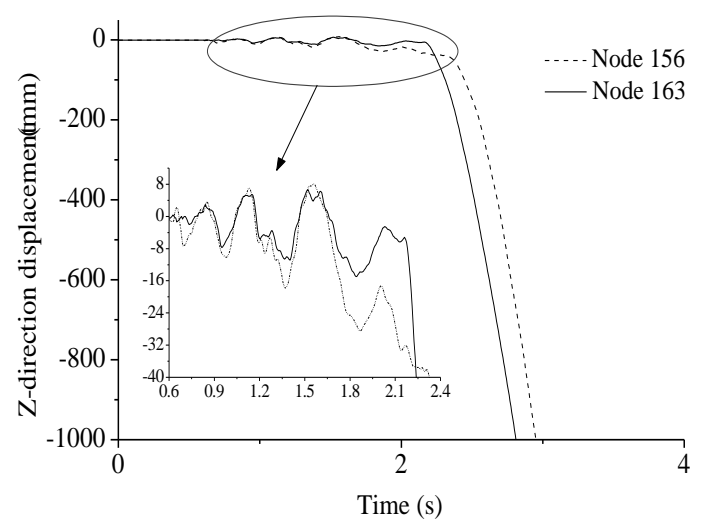

Fig. 15

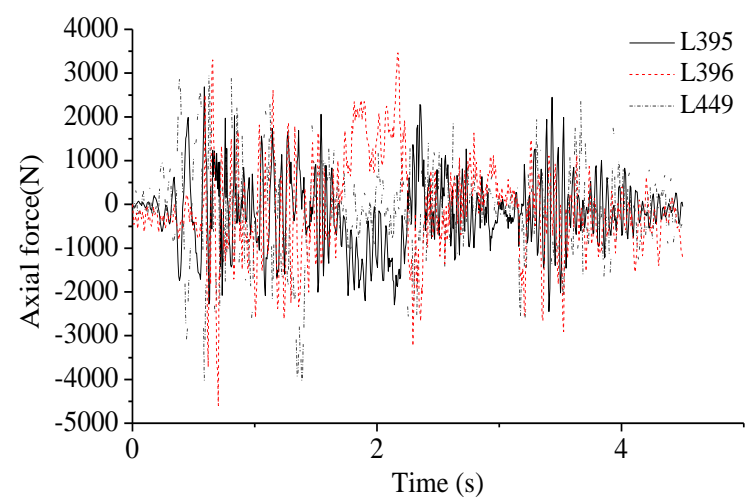

Fig. 16 